Meta

Journal des traducteurs

Translators' Journal

\title{
La traduction vers une langue étrangère : compétences, attitudes, contexte social
}

\section{Tomáš Duběda}

Volume 63, numéro 2, août 2018

URI : https://id.erudit.org/iderudit/1055149ar

DOI : https://doi.org/10.7202/1055149ar

Aller au sommaire du numéro

\section{Éditeur(s)}

Les Presses de l’Université de Montréal

ISSN

0026-0452 (imprimé)

1492-1421 (numérique)

Découvrir la revue

Citer cet article

Duběda, T. (2018). La traduction vers une langue étrangère : compétences, attitudes, contexte social. Meta, 63(2), 492-509.

https://doi.org/10.7202/1055149ar
Résumé de l'article

Nous explorons le problème de la traduction non native qui, malgré les risques qu'elle comporte, est fréquemment pratiquée dans de nombreuses cultures. Le contexte sociologique dans lequel ce type de traduction s'inscrit est appréhendé à travers le concept de " permissivité interculturelle ", qui affecte les destinataires, les commanditaires, les agences aussi bien que les traducteurs. Pour évaluer et structurer les compétences des traducteurs tchèques relatives à la traduction non native, nous avons constitué un échantillon de 160 traductions (40 traducteurs; deux groupes socioprofessionnels : étudiants avancés et professionnels de la traduction ; deux langues étrangères : anglais et français ; deux directions ; deux types de texte : publicitaire et juridique). L'analyse des compétences confirme l'hypothèse de la primauté de la traduction native, tout en indiquant que les textes spécialisés sont davantage compatibles avec la traduction non native que les textes à forte composante expressive. Selon les données recueillies, la qualité des traductions non natives n'augmente pas avec l'ancienneté du traducteur, à la différence des traductions natives. L’impact de l'expérience antérieure avec le type de texte en question reste difficile à évaluer.
Ce document est protégé par la loi sur le droit d'auteur. L’utilisation des services d’Érudit (y compris la reproduction) est assujettie à sa politique d'utilisation que vous pouvez consulter en ligne.

https://apropos.erudit.org/fr/usagers/politique-dutilisation/ 


\title{
La traduction vers une langue étrangère: compétences, attitudes, contexte social
}

\author{
TOMÁŠ DUBĚDA \\ Université Charles, Prague, République tchèque \\ dubeda@ff.cuni.cz
}

\begin{abstract}
RÉSUMÉ
Nous explorons le problème de la traduction non native qui, malgré les risques qu'elle comporte, est fréquemment pratiquée dans de nombreuses cultures. Le contexte sociologique dans lequel ce type de traduction s'inscrit est appréhendé à travers le concept de «permissivité interculturelle», qui affecte les destinataires, les commanditaires, les agences aussi bien que les traducteurs. Pour évaluer et structurer les compétences des traducteurs tchèques relatives à la traduction non native, nous avons constitué un échantillon de 160 traductions (40 traducteurs; deux groupes socioprofessionnels: étudiants avancés et professionnels de la traduction; deux langues étrangères: anglais et français; deux directions; deux types de texte: publicitaire et juridique). L'analyse des compétences confirme l'hypothèse de la primauté de la traduction native, tout en indiquant que les textes spécialisés sont davantage compatibles avec la traduction non native que les textes à forte composante expressive. Selon les données recueillies, la qualité des traductions non natives n'augmente pas avec l'ancienneté du traducteur, à la différence des traductions natives. L'impact de l'expérience antérieure avec le type de texte en question reste difficile à évaluer.
\end{abstract}

\section{ABSTRACT}

We explore the issue of L2 translation, which is frequently practised in many cultures, despite the risks that it involves. The sociological aspects of this type of translation are approached through the prism of "cross-cultural allowance," which affects the addressees, clients, agencies and translators. A sample of 160 translations (40 translators; two cohorts - advanced students and practising translators; two foreign languages - English and French; two directions; two text types - promotional and legal) was used to assess Czech translators' competence in non-native translation. The results of the analysis confirm the assumption that translating into one's mother tongue is optimal and indicate that technical texts are more compatible with $\mathrm{L} 2$ translation than texts of a more expressive nature. According to the collected data, contrary to native translations, the quality of L2 translation does not increase in line with the length of professional practice. The effect of previous experience on the quality of L2 translation for the analysed text types yields controversial results.

\section{RESUMEN}

Investigamos el tema de la traducción inversa, practicada con frecuencia en muchas culturas a pesar de los riesgos que conlleva. Los aspectos sociológicos asociados con esta dirección de traducción son presentados a través del concepto de «permisividad intercultural», la cual influye en los receptores, clientes, agencias y también traductores. Hemos obtenido una muestra de 160 traducciones (efectuadas por 40 traductores divididos en dos grupos - estudiantes avanzados de traducción y profesionales; en dos lenguas extranjeras - inglés y francés; en dos direcciones, dos tipos de texto - publicitario y jurídico) y la hemos utilizado para evaluar las competencias de los traductores checos al traducir a su lengua no materna. Los resultados del análisis han confirmado la hipótesis de que la traducción directa es la modalidad óptima y además indican que los 
textos especializados son más compatibles con la traducción inversa que los textos con elementos expresivos. Según los datos obtenidos, la calidad de la traducción inversa, a diferencia de la traducción directa, no incrementa en función de los años de experiencia profesional. El efecto de la experiencia previa en traducción del tipo textual correspondiente resulta difícil de evaluar.

\section{MOTS CLÉS/KEYWORDS/PALABRAS CLAVE}

traduction non native, qualité de la traduction, sociologie, compétences, tchèque L2 translation, translation quality, sociology, competences, Czech

traducción inversa, calidad de la traducción, sociología, competencias, checo

\section{La directionnalité de la traduction en tant que problème théorique}

Un facteur crucial du processus de traduction est sa directionnalité: si on laisse de côté les cas de bilinguisme équilibré, le traducteur travaille soit vers sa langue A (langue maternelle), soit vers sa langue $B$ (langue étrangère). C'est la première situation qui est jugée optimale, comme le laissent supposer la documentation traductologique et les recommandations professionnelles (Newmark 1988; Recommandation de l'UNESCO sur la protection juridique des traducteurs' ${ }^{1}$ Traduction. Faire les bons choix). De plus, le fait que l'écrasante majorité des écrits théoriques sur la traduction fait entièrement abstraction de la traduction non native peut également être considéré comme une preuve tacite de cette présomption. Toutefois, les contextes où le traducteur doit travailler vers sa langue $B$, en violant le «principe de la langue maternelle» (Thelen 2005: 242), sont plus nombreux qu'on ne pense, du moins dans certaines cultures.

Parmi les théoriciens qui acceptent la traduction non native comme un fait de la civilisation contemporaine, citons en premier lieu Campbell (1998), qui insiste sur l'asymétrie politique et sociale des langues, facteur qui génère des situations où la traduction vers la langue B est inévitable. Selon l'auteur, les compétences des traducteurs dans ce type de traduction méritent d'être étudiées plus en profondeur ${ }^{2}$. Pokorn (2005) arrive à la conclusion que même les œuvres littéraires sont compatibles avec l'idée d'une traduction non native. De leur côté, Snell et Crampton (1989) relèvent que dans la traduction spécialisée, un traducteur non natif maîtrisant le sujet peut être un meilleur choix qu'un traducteur natif. Certains chercheurs n'hésitent pas à appliquer des méthodes empiriques à des échantillons symétriques de traductions natives et non natives (Hurtado Albir 2017). Le statut spécifique de l'anglais comme lingua franca et ses répercussions sur la directionnalité de la traduction sont explorées (Campbell 2005; Taviano 2013), les aspects didactiques de la traduction native le sont aussi (Kelly 2006; Wimmer 2011) de même que la dimension culturelle de cette activité (Prunč 2000).

\section{La traduction non native dans un contexte social}

Dans le cas de la culture tchèque, qui compte environ onze millions de personnes, la traduction non littéraire vers la langue $B$ est un phénomène fréquent: si l'on se limite aux langues d'importance européenne comme l'anglais, le français et l'allemand, on constate que la disponibilité en traducteurs ayant ces langues comme langues maternelles est insuffisante pour satisfaire à tous les besoins dictés par l'administration publique, le tourisme ou le commerce extérieur. Ainsi, selon les statistiques 
recueillies en 2011 par Svoboda ${ }^{3}$ sur le marché tchèque de la traduction, 61\% des traducteurs ou interprètes recensés travaillent dans les deux directions. L'espace public ainsi que le paysage des sites web sont saturés de textes dont la qualité trahit une traduction non native. La traduction vers la langue B fait également partie intégrante des programmes d'études universitaires de traduction (p. ex.: Institut de traductologie: cursus offerts), et, dans le cas des traducteurs assermentés, elle est exigée par la loi (Loi $n^{\circ}$ 36/1967 relative aux experts et interprètes assermentés). Une situation similaire a été décrite pour d'autres pays européens parlant une langue de diffusion limitée, comme la Croatie (Pavlović 2007), le Danemark (Hansen, et al. 1998) ou la Finlande (McAlester 1992) ${ }^{4}$.

À part le problème de la disponibilité en traducteurs natifs, l'intérêt de la traduction non native se fonde également sur des considérations économiques: un traducteur local est d'habitude moins cher qu'un traducteur étranger, et le coût supplémentaire d'une révision native peut être dissuasif pour certains clients. La préférence active ou l'acceptation passive de la traduction non native est également nourrie par la conviction généralement répandue que les compétences du traducteur sont symétriques, qu'il traduise vers sa langue maternelle ou vers une langue étrangère (Beeby Lonsdale 2009) 5 . Il ne faut pas oublier que les destinataires des traductions vers les grandes langues internationales, avec l'anglais en première place, sont non seulement des locuteurs natifs de ces langues, mais le public international au sens large ${ }^{6}$. Enfin, il convient de remarquer que les éventuels défauts des traductions vers une langue $B$ restent souvent cachés: premièrement, les non-professionnels tchèques ne sont pas suffisamment compétents pour juger de la qualité d'une traduction vers une langue internationale, et deuxièmement, une partie importante des destinataires des traductions non littéraires (qu'il s'agisse d'un touriste lisant un menu, d'une entreprise étrangère recevant le courrier de son partenaire tchèque ou d'un ressortissant étranger s'informant sur son permis de séjour), n'étant pas payeurs, n'exigent pas une qualité linguistique impeccable, contents d'avoir été servis dans une langue qu'ils comprennent. Si l'on veut donner une interprétation sociologique à cette situation, on peut avancer l'hypothèse d'une "permissivité interculturelle», qui est d'abord une relation d'échange: pour les langues de diffusion limitée comme le tchèque, la traduction vers les langues internationales, inévitable dans le monde global, est souvent assurée par la communauté linguistique locale, et les destinataires font preuve de tolérance à l'égard de ces traductions, appréciant avant tout leur disponibilité 7 . Une autre lecture est celle d'une relation de pouvoir, dans laquelle la langue cible jouit du statut de langue dominante (pour l'anglais, voir Crystal 2003 ou Campbell 2005), tout en étant «aliénée» par d'autres cultures. Cette permissivité interculturelle est partagée par les principaux acteurs du processus de traduction: les commanditaires tchèques sont permissifs puisqu'ils ne se rendent pas compte des risques intrinsèques d'une traduction non native, les agences de traduction sont permissives puisqu'elles confient les traductions vers des langues étrangères à des traducteurs tchèques, les traducteurs tchèques eux-mêmes sont permissifs puisqu'ils acceptent de traduire vers ces langues, et les destinataires étrangers sont permissifs puisqu'ils acceptent des traductions défectueuses, supposant qu'il n'y a pas de solution de rechange à la traduction non native.

Une question évidente est celle des limites de cette permissivité: un nonprofessionnalisme flagrant est susceptible de nuire à la culture qui le tolère ${ }^{8}$. Les 
moyens qui contribuent à maintenir un niveau acceptable de la permissivité interculturelle incluent la conscience professionnelle des traducteurs ${ }^{9}$, le professionnalisme des agences de traduction et la volonté des clients d'exiger des traductions de qualité adéquate. Dans certains cas, l'activisme du grand public peut également porter ses fruits ${ }^{10}$. Tous ces moyens sont également applicables aux traductions vers la langue maternelle; la spécificité des traductions non natives réside toutefois dans leur moindre visibilité.

\section{L'enquête}

Le problème de la traduction vers la langue B est au cœur d'une enquête qui a été menée en 2016-2017 à l'Institut de traductologie de Prague (République tchèque) et dont les principaux objectifs sont les suivants:

- comparer, pour les paires linguistiques tchèque/anglais et tchèque/français, la qualité objective de la traduction native et non native;

- analyser les attitudes des traducteurs à l'égard de ces deux situations;

- comparer les compétences et attitudes des traducteurs professionnels avec celles des étudiants avancés;

- comparer, pour les deux groupes de traducteurs et les deux directions, la qualité des traductions d'un texte publicitaire et d'un texte juridique.

Quarante traducteurs de langue maternelle tchèque ont été soumis à l'enquête. Dans le groupe des novices, nous avons inclus vingt étudiants de l'Institut de traductologie de Prague, qui ont été testés en deuxième année de Master en traduction, c'est-à-dire peu avant de devenir traducteurs diplômés. Dix d'entre eux traduisaient $\mathrm{du} /$ vers l'anglais et dix du/vers le français. Le groupe des professionnels comportait vingt traducteurs diplômés du même institut ayant exercé la profession de traducteur depuis 8 à 15 ans. Comme dans le cas des novices, la proportion des deux langues était égale. Tous les traducteurs pratiquaient la traduction non native d'une manière ou d'une autre, soit dans le cadre de leurs études universitaires, soit dans leur profession.

Chacun des traducteurs testés a traduit, dans des conditions contrôlées, quatre textes d'une longueur d'environ 250 mots, pleinement réalistes au vu de ce que propose le marché tchèque de la traduction:

- deux textes publicitaires (présentations d'entreprises) de difficulté comparable, l'un vers sa langue maternelle, et l'autre vers la langue étrangère;

- deux textes juridiques (conditions d'utilisation d'un site web) de difficulté comparable, l'un vers sa langue maternelle, et l'autre vers la langue étrangère.

Les textes, authentiques, ont été raccourcis afin d'avoir une longueur et une difficulté comparables. Le processus de sélection des textes de départ était collectif: les trois chercheurs impliqués dans le projet ont vérifié que la présence des principaux éléments déterminant la difficulté du texte (terminologie spécialisée, lexique concret/ abstrait, complexité de la syntaxe, références culturelles, éléments expressifs...) soit comparable. Dans le cas du texte juridique, nous avons procédé à une calibration plus fine, dont le but était d'arriver à un nombre identique ou quasi identique de termes juridiques univerbaux, de termes juridiques multiverbaux et de clichés phrastiques typiques de la langue de droit. Trois extraits des textes utilisés se trouvent en annexe du présent article. 
Les traducteurs travaillaient à leur domicile et pouvaient se servir de dictionnaires et d'autres ressources imprimées, électroniques ou en ligne. Ils disposaient de 90 minutes pour la traduction de chacun des textes. Dans les instructions données aux traducteurs, nous avons précisé que la traduction était censée être publiée sur le web de la société commanditaire, pour s'adresser à la clientèle tchécophone de celle-ci.

Chaque traduction vers le tchèque a été évaluée par un groupe de trois experts tchèques (dont l'auteur du présent article), et chaque traduction vers la langue étrangère, par deux experts de langue maternelle anglaise ou française (ressortissants britanniques et français, respectivement). Tous les évaluateurs sont des traducteurs professionnels ou des enseignants en traduction. L'évaluation s'est faite selon un protocole rigoureux, et ce, parallèlement à deux niveaux (Martínez Mateo 2014; O'Brien 2012) : l'évaluation locale consistait à identifier et classifier les fautes de traduction, et l'évaluation globale, à attribuer à chaque traduction une note globale. L'échelle des notes est définie de façon suivante:

- A: traduction excellente, pleinement conforme aux standards d'une traduction professionnelle, avec, éventuellement, un petit nombre de révisions nécessaires.

- B: traduction correcte, plus ou moins conforme aux standards d'une traduction professionnelle, avec un certain nombre de révisions nécessaires.

- C: traduction passable, en partie conforme aux standards d'une traduction professionnelle, mais nécessitant une révision approfondie.

- F: traduction inacceptable, nécessitant une révision substantielle pour atteindre un niveau professionnel.

Malgré l'effort d'assurer, à travers des règles soigneusement formulées, un niveau de cohérence aussi élevé que possible, l'évaluation est nécessairement empreinte de subjectivité. En effet, la qualité d'une traduction étant un concept relatif (Mossop $2001)^{11}$, chaque évaluateur applique sa propre idée de ce qu'est une «traduction professionnelle» (c'est-à-dire une traduction conforme aux règles de l'art), terme qui sert de point de référence central dans notre système d'évaluation (voir l'échelle des notes plus haut). Le recours à des binômes d'experts (dans le cas des traductions non natives) ou à un groupe de trois experts (dans le cas des traductions natives) contribue à estomper les effets de cette subjectivité.

L'accord entre les évaluateurs, exprimé par le coefficient de corrélation ${ }^{12}$, est de 0,82 pour les traductions vers le tchèque (forte corrélation positive), et de 0,48 pour les traductions vers la langue étrangère (faible corrélation positive) ${ }^{13}$. Calculés séparément pour les deux langues, les coefficients de corrélation entre les notes assignées aux traductions non natives sont de 0,48 pour l'anglais et de 0,42 pour le français. $\grave{A}$ part les convictions personnelles sur la qualité des traductions, la différence qu'on observe dans les niveaux d'accord s'explique également par le fait que les évaluateurs des traductions vers le tchèque avaient eu l'occasion de faire une analyse collective des textes de départ, alors que cette même analyse n'était pas possible dans le cas des évaluateurs étrangers, dont certains ne vivaient pas en République tchèque. Quoique l'évaluation même soit restée individuelle, cette analyse préalable a probablement augmenté le niveau d'accord des évaluateurs tchèques, et constitue une certaine faiblesse de notre méthode. Un autre facteur qui pourrait jouer un rôle est lié à la typologie des fautes qui, dans leur ensemble, déterminent la note finale: si les deux directions de traduction peuvent produire en principe le même répertoire de fautes (sens, terminologie, grammaire, style, etc.), la direction vers une langue étrangère est 
spécifique en ce qu'elle comporte différentes manifestations d'«étrangeté», dont l'évaluation laisse un espace supplémentaire aux jugements subjectifs. De plus, évaluer un texte rédigé par un étranger est une tâche qui peut mobiliser des réflexions d'ordre interculturel, voire moral (notion de "permissivité interculturelle» évoquée plus haut).

Au cours de l'enquête, chaque traducteur avait à remplir trois questionnaires: le premier concernait son profil de traducteur, le deuxième comportait des questions axées sur les deux textes publicitaires qu'il venait de traduire, et le troisième, des questions sur les textes juridiques. Les questionnaires sont donnés, dans une forme abrégée, en annexe. Pour chaque traduction, nous avons noté le temps de travail, qui ne devait pas dépasser 90 minutes.

\section{Objectif et hypothèses}

La présente étude se propose de faire une comparaison qualitative des traductions vers la langue B avec les traductions vers la langue A et d'analyser l'influence d'un ensemble de facteurs relevant du profil du traducteur et de son ressenti, dans le but d'en dégager des paramètres de ce que nous avons appelé plus haut la "permissivité interculturelle». Aux fins de notre analyse, nous faisons abstraction de la typologie des erreurs, prenant en compte uniquement la qualité globale des traductions telle qu'elle est exprimée par les notes d'ensemble assignées par les évaluateurs. En effet, une analyse systématique des fautes identifiées dans notre échantillon serait trop ample pour être intégrée dans le présent texte ${ }^{14}$. Aussi ne citons-nous pas d'exemples, à l'exception des extraits en annexe.

Les six hypothèses que nous entendons vérifier sont les suivantes:

- 1) Hypothèse de la primauté de la traduction vers la langue A. La qualité globale des traductions vers la langue $\mathrm{B}$ sera inférieure à celle des traductions vers la langue $\mathrm{A}$ (Newmark 1988).

- 2) Hypothèse de la symétrie des compétences. La qualité des traductions natives et non natives sera corrélée: plus les compétences d'un traducteur vers la langue A sont bonnes, mieux il traduit vers la langue $\mathrm{B}$, et vice versa.

- 3) Hypothèse de l'effort stylistique. La qualité globale des traductions vers la langue B sera inférieure pour les textes publicitaires, qui nécessitent une bonne maîtrise du style. En revanche, elle sera supérieure pour les textes juridiques, plus formalisés (Snell et Crampton 1989).

- 4) Hypothèse de l'impact positif de l'expérience professionnelle. L'expérience professionnelle (étudiants en traduction vs traducteurs professionnels, pratique de la traduction non native, degré de connaissance des genres en question) se reflétera dans la qualité des deux types de traductions.

- 5) Hypothèse de la dominance de l'anglais. Le statut particulier de l'anglais en République tchèque et, plus généralement, en Europe (langue globale, langue étrangère la plus enseignée, première langue étrangère sur le marché de la traduction) se traduira par une meilleure qualité des traductions vers l'anglais.

- 6) Hypothèse de la capacité d'autoévaluation. La satisfaction subjective du traducteur, telle qu'elle est exprimée dans les questionnaires, sera positivement corrélée avec la qualité objective de sa prestation.

Certaines de ces hypothèses semblent évidentes, car elles correspondent aux intuitions généralement partagées par les théoriciens comme les praticiens. Toutefois, 
pour qu'elles puissent être scientifiquement fondées et aspirer à un statut de «loi du comportement traductionnel» dans le sens de Toury (1995), il est nécessaire de les soumettre à une validation empirique.

\section{Résultats}

\subsection{Hypothèse de la primauté de la traduction vers la langue A}

La Figure 1 montre la répartition des notes assignées par les réviseurs.

FIGURE 1

Distribution des notes pour les deux types de traduction : 240 notes ont été attribuées aux traductions vers la langue $A$ (40 traducteurs $\times 2$ textes $\times 3$ réviseurs), et 160 notes aux traductions vers la langue $B$ (40 traducteurs $x 2$ textes $x 2$ réviseurs).

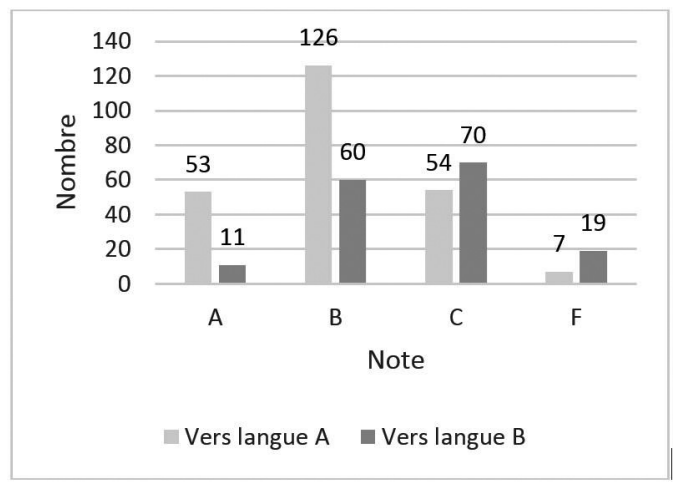

La note la plus fréquente pour les traductions natives est B; pour les traductions non natives, ce sont les notes $\mathrm{B}$ et $\mathrm{C}$ qui prédominent. En convertissant l'échelle des notes $\mathrm{A}-\mathrm{B}-\mathrm{C}-\mathrm{F}$ en valeurs numériques $1-2-3-4$, nous arrivons à une moyenne de $2,0(\mathrm{~B})$ pour les traductions vers la langue maternelle, et à une valeur de 2,6 $(\mathrm{C}+)^{15}$ pour les traductions vers la langue étrangère. Les deux distributions sont monomodales et la différence de leurs moyennes est statistiquement significative ${ }^{16}$. Vingt-deux pour cent des traductions natives ont été notées A, contre sept pour cent des traductions non natives. La note $\mathrm{F}$ a été plus fréquemment attribuée aux traductions non natives $(12 \%)$ qu'aux traductions natives $(3 \%)$.

Ces chiffres confirment la difficulté de la traduction non native: une qualité excellente (A) n'est atteinte que rarement, alors qu'une traduction sur huit est de qualité inacceptable $(\mathrm{F})$.

La difficulté intrinsèque de la traduction vers la langue $\mathrm{B}$ a été également confirmée subjectivement (questionnaire 1, question 4 - voir annexe) : 32 traducteurs sur 40 considèrent ce type de traduction comme plus difficile que la traduction vers leur langue maternelle. Deux traducteurs trouvent que la traduction non native est moins difficile, et six traducteurs estiment que la difficulté est comparable dans les deux directions. La traduction non native se montre également comme plus exigeante en termes de temps de travail: les traductions vers le tchèque ont été remises en moyenne après 76 minutes, et les traductions vers l'anglais ou vers le français, après 80 minutes. La différence, sans être importante, est statistiquement significative ${ }^{17}$. 


\subsection{Hypothèse de la symétrie des compétences}

À supposer que la compétence des traducteurs soit équilibrée, la qualité relative des traductions vers la langue A devrait être comparable, pour un même traducteur, à la qualité relative de ses traductions vers la langue $\mathrm{B}^{18}$. Ceci n'a été confirmé que partiellement par nos données: le coefficient de corrélation entre la qualité des traductions natives et non natives ${ }^{19}$ est de 0,25 , ce qui correspond à une corrélation positive, mais assez faible.

Comme il a été dit plus haut, les notes assignées aux traductions non natives sont, dans l'ensemble, inférieures à celles assignées aux traductions natives. Dans ce contexte, il serait intéressant d'examiner de plus près les traducteurs qui ont obtenu une meilleure note pour la traduction non native que pour la traduction native. Si l'on fait la comparaison de la note moyenne, on trouve parmi les 80 paires de textes (40 traducteurs $\mathrm{x} 2$ types de textes) 18 cas où la valeur est meilleure pour la traduction vers la langue B. Il est frappant que ce groupe de traductions «à qualité inverse» comprenne 14 traductions juridiques, et seulement 3 traductions publicitaires: cette observation apporte une confirmation supplémentaire de l'hypothèse de l'effort stylistique, traitée plus bas.

\subsection{Hypothèse de l'effort stylistique}

Il semble plausible de postuler que l'acceptabilité des traductions produites par des traducteurs non natifs dépende, plus que chez les traducteurs natifs, du type de texte. Lors de la traduction d'un texte informatif, formulé dans un langage peu expressif, le traducteur mobilise avant tout des moyens linguistiques conventionnels, tout en ayant à sa disposition plusieurs types de ressources qui permettent de contourner les risques liés à la traduction non native (dictionnaires spécialisés, textes comparables dans la langue d'arrivée, corpus linguistiques). Les textes à forte composante expressive (textes littéraires, essais, textes publicitaires), en revanche, nécessitent une maîtrise plus fine du style, afin de trouver le juste ton et de bien reproduire les fonctions expressive, poétique et conative du texte. Le recours à des ressources lexicologiques ou textuelles est bien moins efficace dans ce cas. Ainsi, certains auteurs sont d'avis que c'est dans la traduction spécialisée que les traducteurs non natifs sont susceptibles de produire des textes de qualité acceptable (Snell et Crampton 1989; Beeby Lonsdale 2009) ${ }^{20}$.

Nous avons comparé, dans le groupe des traductions non natives, les notes en fonction du type de texte (Figure 2). Les textes publicitaires (note moyenne de 2,9, c'est-à-dire $\mathrm{C}$ ) sont évalués comme moins acceptables que les textes juridiques (note moyenne de 2,4, c'est-à-dire B-). Cette différence, égale à une demi-note, est statistiquement significative ${ }^{21}$. À titre de comparaison, la notation des deux genres textuels est similaire (autour de B) dans le cas des traductions vers la langue maternelle (texte publicitaire: 2,0 ; texte juridique: 2,1$)^{22}$.

Il convient cependant de préciser que les résultats observés peuvent être partiellement biaisés par les différences entre les attentes des évaluateurs. Les personnes chargées d'évaluer les traductions vers l'anglais et le français sont des traducteurs ou enseignants en traduction expérimentés, mais sans spécialisation dans le domaine juridique. En revanche, deux des trois évaluateurs des traductions vers le tchèque 
FIGURE 2

Notes moyennes pour les deux types de textes

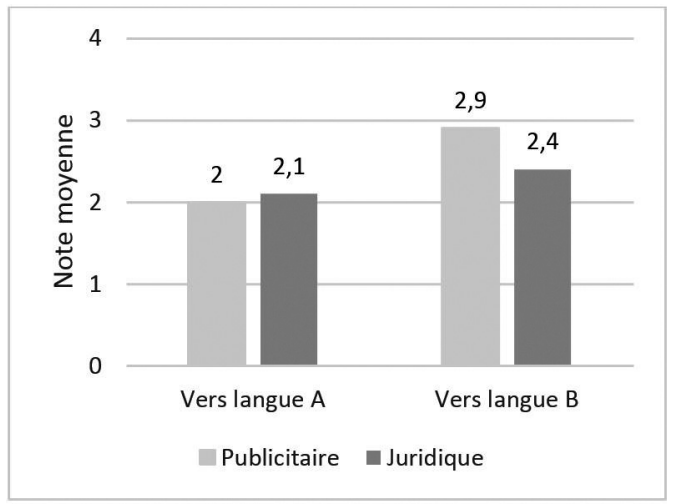

sont des enseignants en traduction juridique et des praticiens de ce type de traduction. Ainsi, la différence d'une demi-note que nous avons constatée dans les traductions non natives traduit certes une compétence textuelle inégale, mais il est possible qu'avec un niveau d'exigences plus élevé sur le plan de la terminologie et de la phraséologie juridiques, cette différence soit moindre.

\subsection{Hypothèse de l'impact positif de l'expérience professionnelle}

Nos données laissent supposer que la différence entre professionnels et étudiants se reflète dans la qualité des traductions vers la langue $\mathrm{A}$ : la note moyenne des professionnels est de 1,7 (B+), et celle des étudiants, de 2,4 (B-) ${ }^{23}$. Pour les traductions non natives, la variable reste sans effet: les deux notes sont très proches (professionnels: 2,6, étudiants: 2,7 , c'est-à-dire $\mathrm{C}+$ ) et leur différence n'est pas statistiquement significative ${ }^{24}$.

FIGURE 3

Notes moyennes pour les deux groupes de traducteurs

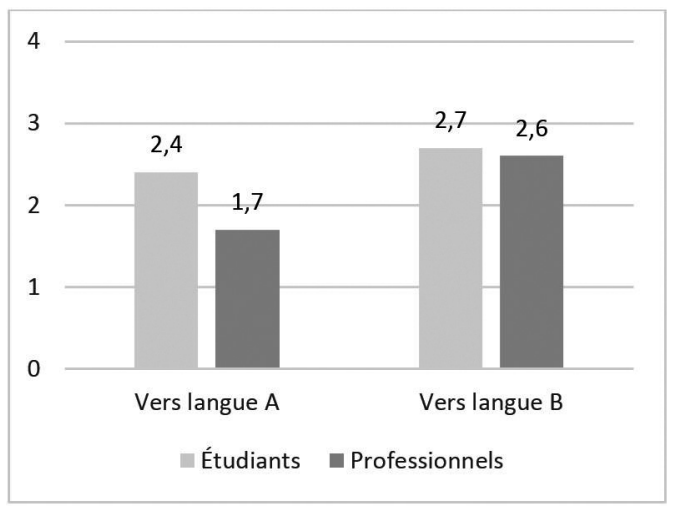


Il semble contre-intuitif que la différence entre les deux groupes testés ne se reflète pas dans la qualité des traductions non natives. Une première explication possible serait l'existence d'un plafond de compétences, qui serait atteint à la fin des études supérieures, et qui serait difficile à franchir malgré la pratique régulière de la traduction non native. Ce plafond correspondrait grosso modo à la note C. Une deuxième explication serait d'ordre diachronique: le fait que les professionnels d'aujourd'hui atteignent les mêmes résultats que les futurs diplômés peut s'expliquer par l'évolution du niveau des études supérieures, du bagage linguistique des étudiants et de l'exposition générale aux langues mondiales. Les diplômés d'il y a une décennie auraient atteint, grâce à la pratique, un niveau que les diplômés actuels ont aujourd'hui. Cette hypothèse impliquerait, d'une part, que le niveau des étudiants peut encore évoluer, et d'autre part, que le niveau moyen des professionnels était proche de la note C ou inférieur à celle-ci lors de leurs premiers pas dans le monde professionnel. Nous penchons intuitivement pour la première explication, sans toutefois pouvoir trancher la question à l'aide des données dont nous disposons.

Dans un deuxième temps, nous avons mis en relation la qualité des traductions vers l'anglais ou le français avec la part respective des traductions natives et non natives dans la pratique professionnelle des traducteurs professionnels. Les étudiants, n'ayant pas de véritable expérience professionnelle, ont été exclus de cette partie de l'analyse. Dans le questionnaire 1 (question 3 - voir annexe), les participants ont indiqué le volume approximatif de traductions vers la langue $\mathrm{B}$ dans leur activité professionnelle $(0 \text { à } 20 \% ; 21 \text { à } 40 \% ; 41 \text { à } 60 \% ; 61 \text { à } 80 \% ; 81 \text { à } 100 \%)^{25}$. Après avoir converti ces intervalles en valeurs numériques $(1 ; 2 ; 3 ; 4 ; 5)$, nous avons établi, pour chaque traducteur, le coefficient de corrélation entre cette valeur et la note moyenne obtenue pour les deux traductions vers la langue étrangère: le résultat obtenu est de $r=0,05$. Ce chiffre exprime une corrélation négative (la meilleure note étant 1 , et non pas 4 ), mais négligeable: il n'y aurait donc pas d'effet du volume de travail vers la langue B sur la qualité de ces traductions. Calculée séparément pour les traductions publicitaires et juridiques, la valeur du coefficient de corrélation reste également très basse.

Troisièmement, nous nous sommes intéressé à la fréquence avec laquelle le traducteur rencontre, dans sa vie professionnelle, le type de texte en question. Comme plus haut, cette analyse a été limitée aux professionnels. La question correspondante des questionnaires 2 et 3 (question 6 - voir annexe), posée indépendamment pour chacune des directions de traduction, permettait quatre réponses: jamais; quasiment jamais; parfois; souvent. Ces réponses ont été converties en valeurs numériques $(0$; $1 ; 2 ; 3)^{26}$ puis corrélées avec la note moyenne obtenue. Comme en témoigne la Figure 4, la seule situation où l'influence de la connaissance du genre semble être confirmée est la traduction du texte publicitaire vers le tchèque: ici, le coefficient de corrélation $(\mathrm{r}=-0,26)$ correspond à une faible corrélation positive (en dépit de la valeur négative, causée par l'orientation inverse de l'échelle des notes). Pour les traductions juridiques vers le tchèque, la valeur du coefficient $(\mathrm{r}=0,31)$ traduit une faible corrélation négative: plus l'expérience est importante, moins la note est bonne. Cette observation, qui n'est pas sans surprendre, peut être due soit au hasard, soit à l'effet de fossilisation (traduction stéréotypée, sans vérification systématique des équivalents, avec une capacité d'autocritique estompée). Quant à la traduction vers la langue B, la valeur du coefficient est proche du zéro pour les deux types de textes. Il n'y aurait donc pas d'effet observable de la connaissance du genre sur la qualité de la traduction non native. 
FIGURE 4

Coefficients de corrélation entre la note moyenne et la connaissance du genre.

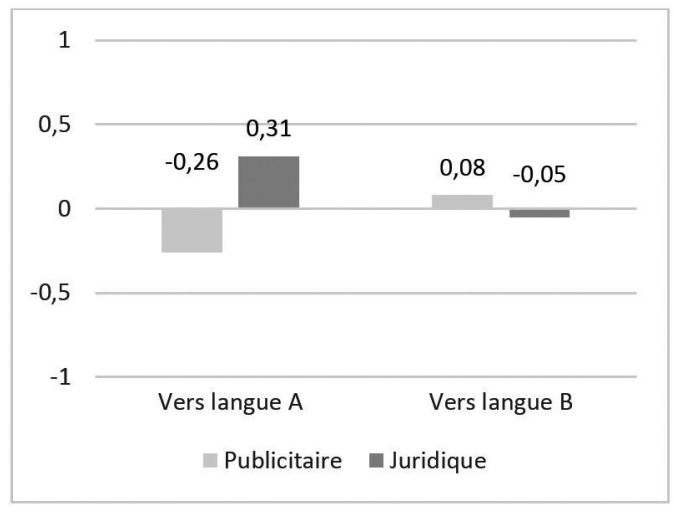

Pour les traductions juridiques réalisées par les professionnels, nous avons enfin testé un quatrième aspect, qui peut être également rapproché de la catégorie de l'expérience professionnelle, à savoir le niveau de compétences dans le domaine du droit, qui est un facteur clé de la qualité de la traduction juridique (Prieto Ramos 2015). Dans la question 16 du questionnaire 1 (voir annexe), les traducteurs sont invités à préciser quelles formations ils ont suivies en dehors du cadre universitaire. Sur les 20 professionnels soumis à l'enquête, 12 ont déclaré avoir suivi un cours de droit et/ou de traduction juridique. La note moyenne obtenue pour la traduction juridique dans ce groupe est de 1,9 (B) dans le cas de la traduction native et de 2,5 $(\mathrm{C}+)$ dans le cas de la traduction non native, contre 1,5 (B+) et 2,1 (B) dans le groupe des traducteurs n'ayant pas suivi une telle formation. Dans les deux cas donc, on est en présence d'un résultat paradoxal, et ce, d'autant plus que la différence constatée est statistiquement significative ${ }^{27}$. Rappelons cependant que ce résultat, difficilement explicable, est basé sur un nombre limité d'observations (professionnels uniquement, textes juridiques uniquement) ${ }^{28}$.

\subsection{Hypothèse de la dominance de l'anglais}

Si l'on compare la moyenne des notes assignées aux traductions vers l'anglais $(2,4$, c'est-à-dire $\mathrm{B}-)$ et vers le français $(2,8$, c'est-à-dire $\mathrm{C}+)$, on constate une légère asymétrie ${ }^{29}$; celle-ci apparaît également lorsqu'on fait la comparaison séparément pour les deux types de textes et pour les deux groupes de traducteurs (étudiants vs professionnels). Deux faits permettent d'expliquer cette asymétrie: premièrement, la maîtrise de l'anglais parmi les traducteurs et les étudiants est probablement supérieure à celle du français, et, deuxièmement, les attentes stylistiques des évaluateurs français peuvent être plus élevées que celles des évaluateurs britanniques (Mossop 2001; O’Brien 2012).

\subsection{Hypothèse de la capacité d'autoévaluation}

Une des questions de l'enquête portait sur la satisfaction du traducteur quant à la traduction qu'il venait d'effectuer, et ce, séparément pour les deux directions 
(Questionnaires 2 et 3, question 7 - voir annexe). Les participants pouvaient choisir entre cinq réponses: très content(e); plutôt content(e); ni content(e) ni mécontent(e); plutôt mécontent(e); très mécontent(e). Ces réponses, une fois converties en valeurs numériques $(-2 ;-1 ; 0 ; 1 ; 2)$, ont été corrélées avec la note objective. Comme le montre la Figure 5, la capacité d'auto-évaluation s'est révélée dans l'ensemble faible (les coefficients négatifs traduisent en l'occurrence une corrélation positive). Dans le cas de la traduction publicitaire vers l'anglais ou vers le français, la corrélation est négligeable (valeur proche du zéro). Si l'on accepte l'idée que cette différence a un fondement réel, on pourrait soulever l'hypothèse qu'il s'agit de l'effet conjoint de la traduction non native et de la difficulté stylistique du texte qui fait que les traducteurs sont incapables de porter un regard objectif sur leurs prestations. Ces résultats relativisent quelque peu l'hypothèse de Mossop (2001) selon laquelle la capacité de juger de la qualité de ses propres traductions devrait être considérée comme faisant partie de la compétence du traducteur.

FIGURE 5

Coefficients de corrélation entre la note moyenne et la satisfaction subjective des traducteurs

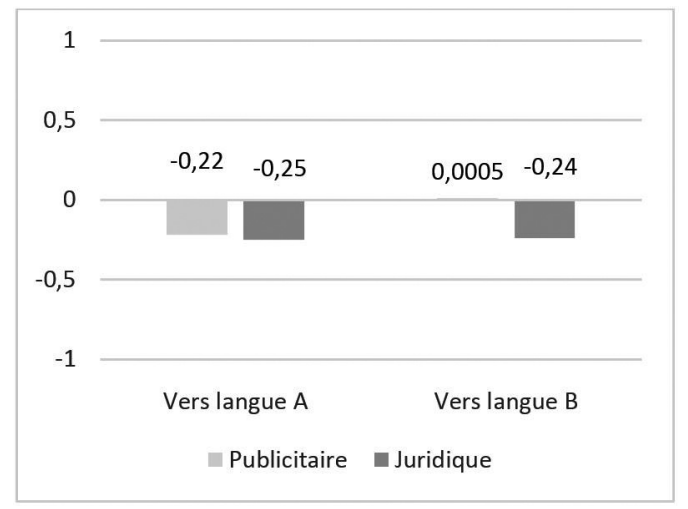

\section{Discussion et conclusions}

Avant de procéder à une synthèse de nos observations, nous jugeons utile de rappeler plusieurs aspects méthodologiques qui doivent être pris en compte lors de l'interprétation des résultats et qui pèsent sur la validité de nos conclusions. Premièrement, quoique l'étude se veuille réaliste en ce sens que nous avons tâché de créer des conditions proches du quotidien des traducteurs professionnels, notamment en termes de genres textuels et d'outils de travail, nos traducteurs n'avaient pas la possibilité de refuser les textes à traduire, décision que certains d'entre eux auraient peut-être prise dans une situation réelle. De plus, l'ensemble de nos analyses se rapportent aux traductions dans leur forme brute, c'est-à-dire avant révision, ce qui ne nous permet pas d'émettre des conclusions directes sur l'état du marché de la traduction en République tchèque. Deuxièmement, malgré notre volonté d'assurer une comparabilité aussi élevée que possible entre les différents sous-ensembles de la base textuelle (difficulté calibrée des textes, groupes homogènes de traducteurs, profils comparables des évaluateurs), nous ne pouvons pas exclure des idiosyncrasies tant sur le plan des textes utilisés que sur le plan des évaluateurs. Le traitement statistique 
basé sur des moyennes (moyenne des notes assignées par deux ou trois évaluateurs, moyenne des notes pour les deux types de textes, etc.) rend toutefois les conclusions plus robustes et permet d'estomper l'effet des variables parasites. Troisièmement, les quarante sujets testés, qui ont tous suivi des études de traduction à l'Institut de traductologie de Prague, ne couvrent certainement pas toute l'étendue du marché de la traduction en République tchèque. Nos conclusions sont ainsi une espèce de diagnostic institutionnel, représentatif d'un segment de marché que l'on pourrait peut-être qualifier d'«idéal».

L'analyse qui fait l'objet de cet article a tout d'abord permis de confirmer l'hypothèse de la primauté de la traduction native: pour les traducteurs diplômés de nationalité tchèque, la traduction vers une langue internationale est moins aisée que la traduction vers leur langue maternelle et se traduit par des résultats de qualité inférieure. La note moyenne est $\mathrm{B}$ pour les traductions natives, et $\mathrm{C}+$ pour les traductions non natives. Ceci ouvre la question de l'utilisabilité pratique des traductions non natives: sans révision par un locuteur natif, l'usage des textes traduits vers la langue B devrait rester limité, tout au plus, aux contextes informels. Dans le cas des traductions notées $\mathrm{F}$, qui représentent $12 \%$ des textes dans cette catégorie, la révision peut s'avérer non rentable. Rares sont les traducteurs qui ont obtenu la note A pour leur traduction vers l'anglais ou le français et qui, si leur niveau de compétences s'avère stable, peuvent aspirer au statut de professionnels hautement compétents, dont la production en langue étrangère ne nécessite qu'une révision minimale. Cependant, sur une échelle à quatre niveaux de qualité $(\mathrm{A}-\mathrm{B}-\mathrm{C}-\mathrm{F})$, la différence d'une demi-note (B pour les traductions natives et $\mathrm{C}+$ pour les traductions non natives) ne parait pas dramatique. Nos résultats rejoignent, grosso modo, les conclusions du projet d'évaluation des compétences en traduction mené par le groupe PACTE (Hurtado Albir 2017), dans lequel les traducteurs professionnels ont obtenu un score moyen de 0,73 pour la traduction native et de 0,52 pour la traduction non native (sur une échelle allant de 0 à 1$)^{30}$.

La deuxième hypothèse, concernant la corrélation qualitative entre traduction native et traduction non native, n'a été confirmée que partiellement: cette corrélation est faible, ce qui parle en faveur de l'existence de différents profils de traducteurs en termes de symétrie de compétences.

L'hypothèse de l'effort stylistique, selon laquelle la capacité des traducteurs non natifs à produire des textes acceptables devrait être meilleure pour le genre juridique que pour le genre publicitaire, a été confirmée: alors que la note moyenne des traductions vers le tchèque est presque identique (B), les traductions non natives sont notées $\mathrm{B}$ - et $\mathrm{C}$, respectivement.

L'hypothèse de l'impact positif de l'expérience professionnelle a été subdivisée en quatre volets. Une première constatation est que les traducteurs diplômés exerçant leur métier depuis 8 à 15 ans ont réalisé des traductions natives de qualité significativement supérieure à celles réalisées par les étudiants en $2^{\mathrm{e}}$ année de Master (notes $\mathrm{B}+$ et $\mathrm{B}-$, respectivement). En ce qui concerne les traductions non natives, en revanche, l'ancienneté n'a pas d'effet visible sur la note moyenne. Pour expliquer cette observation, qui va à l'encontre de ce qui est généralement admis, nous avons proposé deux hypothèses: celle du plafond des compétences et celle de l'évolution diachronique du niveau des études universitaires. Quant au volume de traductions vers la langue B dans la pratique professionnelle du traducteur (testé uniquement pour les 
professionnels), nous avons vu que celui-ci n'a aucun effet tangible sur la qualité des traductions. L'exposition antérieure aux traductions de même type, quant à elle, se traduit de manière variable. Plus spécifiquement, dans le domaine de la traduction juridique, la formation en droit s'est montrée - et c'est là le résultat le plus surprenant de notre enquête - un facteur globalement défavorable. En somme, les observations dans la catégorie "expérience professionnelle» sont en désaccord avec l'hypothèse formulée, soulevant certaines questions au regard des capacités d'apprentissage et d'évolution professionnelle.

La qualité des traductions vers l'anglais s'est montrée supérieure à la qualité des traductions vers le français, corroborant ainsi la cinquième hypothèse. L'anglais semble devancer le français (et, par extension, les autres langues étrangères sur le marché tchèque des traductions) sur un plan quantitatif comme qualitatif.

La dernière hypothèse postulait que les traducteurs sont conscients du niveau de leurs compétences. À la lumière de nos résultats, nous pouvons constater que la capacité d'auto-évaluation est faible: les jugements exprimés dans les questionnaires semblent calibrés subjectivement, par rapport à la performance individuelle de chaque traducteur, et non pas par rapport à un niveau de référence généralement admis.

Le but de notre projet a été d'apporter des données empiriques relatives à la compétence traductive des traducteurs non natifs, données qui permettent de mieux comprendre la sociologie de la traduction et qui peuvent orienter les choix dans le milieu professionnel. Nos constatations s'inscrivent dans la logique de la "permissivité interculturelle» que nous avons caractérisée au début de l'article: la pratique de la traduction non native est perçue comme exigeante mais légitime; elle fournit des produits qui, dans leur forme brute, ne sont pas prêts à être mis en circulation, mais qui, une fois révisés par un locuteur natif, peuvent le devenir.

\section{REMERCIEMENTS}

Le présent article a bénéficié du soutien du projet GAČR 16-03037S Directionnalité de la traduction: aspects qualitatifs et sociologiques. Lauteur remercie les deux relecteurs anonymes de leurs suggestions.

\section{NOTES}

1. «Le traducteur doit, dans la mesure du possible, traduire dans sa langue maternelle ou dans une langue qu'il possède comme sa langue maternelle» (Article 14d).

2. «The translator can no longer be thought of as a ghostly perfect bilingual, but as a living being with a role and abilities that can be described and discussed...» (p. 4).

3. Svовода, Tomáš (2011): Průzkum překladatelského trhu [Enquête sur le marché de la traduction]. Tlumočení a překlad (ToP). 99:15-17. La version longue de cette enquête peut être consultée à l'adresse suivante : <http://www.jtpunion.org/O-JTP/Clanky-a-zajimavosti/ANKETA-PRUZKUMPREKLADATELSKEHOTRHU>.

4. Il s'avère que la pratique de la traduction non native est systématique également dans certaines grandes cultures européennes (Roiss 2001) ou non européennes (Baorong 2009).

5. «In popular belief, linguistic comptence is symmetrical: the general public makes no distinction between translating from a foreign language into a mother tongue and vice versa and assumes that a translator will have no difficulty translating in both directions. This belief often extends to employers» (p. 64).

6. «We would argue that the view that translators should work into their mother tongue is a meme which is fast becoming unenforceable and impractical in this era of globalised communications and intercultural exchanges» (Adab 2005: 227). 
7. «In most cases a less-than-perfect translation is better than none at all» (Wagner 2011: 225).

8. Un cas visible du non-professionnalisme de l'administration publique est la version française du Code civil tchèque, publiée récemment par le ministère de la Justice.

9. Un des traducteurs contactés dans le cadre de notre enquête a refusé d'y participer, expliquant qu'il ne traduisait vers la langue étrangère que dans des cas exceptionnels et avec beaucoup de scrupules, alors qu'il a de bonnes raisons de croire qu'il produirait une traduction dont la qualité serait supérieure à ce qui est la norme sur le marché des traductions non natives.

10. L'auteur a dans son entourage des personnes de langue maternelle anglaise ou française vivant à Prague qui ont signalé des défauts de traduction dans l'espace public; dans deux cas au moins, ceci a mené à une amélioration.

11. "There is no such thing as absolute quality» (Mossop 2001: 6).

12. Le coefficient de corrélation Pearson a été calculé, dans le cas des traductions vers l'anglais ou le français, entre les notes assignées par chacun des deux évaluateurs. Dans le cas des traductions vers le tchèque, où il $\mathrm{y}$ avait trois évaluateurs, la valeur finale a été établie comme moyenne des coefficients calculés pour chaque paire d'évaluateurs.

13. Grosjean et Dommergues (2011).

14. Les stratégies de traduction et les équivalents choisis sont analysés p. ex. dans Obdržálková (2016) ou Duběda et Obdržálková (2018).

15. Pour faciliter l'interprétation des notes, les nombres décimaux sont convertis en lettres de façon suivante: 1,0 à $1,1(\mathrm{~A}) ; 1,2$ à $1,4(\mathrm{~A}-) ; 1,5$ à $1,8(\mathrm{~B}+) ; 1,9$ à $2,1(\mathrm{~B})$ et ainsi de suite.

16. T-test unilatéral $(\mathrm{p}<0,001)$.

17. T-test unilatéral $(\mathrm{p}<0,001)$.

18. Par «qualité relative», nous entendons la qualité absolue ramenée à la moyenne. La moyenne est de 2,0 pour la traduction native et de 2,6 pour la traduction non native (cf. partie 5.2).

19. Le coefficient de corrélation Pearson a été calculé pour la moyenne des notes attribuées à chaque traduction.

20. «Given access to sufficient documentation, conscientious inverse translators can produce competent translations of the standardized discourse fields which are common in business, science, technology and public administration» (Beeby Lonsdale 2009: 66).

21. T-test unilatéral $(\mathrm{p}<0,001)$.

22. Cette différence n'est pas statistiquement significative (t-test unilatéral, $p=0,25$ ).

23. Cette différence est statistiquement significative (t-test unilatéral, $\mathrm{p}<0,001$ ).

24. T-test unilatéral $(\mathrm{p}=0,23)$.

25. La répartition des réponses à cette question est: $5 ; 6 ; 7 ; 1 ; 1$.

26. La moyenne des réponses est de 1,2 pour les textes publicitaires vers le tchèque, de 1,2 pour les textes publicitaires vers la langue étrangère, de 1,7 pour les textes juridiques vers le tchèque, et de 1,6 pour les textes juridiques vers la langue étrangère.

27. T-test unilatéral ( $\mathrm{p}=0,004$ et 0,01 , respectivement).

28. Dans Duběda et Obdržálková (2018), nous avons entrepris une analyse approfondie des équivalents terminologiques. Cette analyse, qui concerne uniquement la traduction juridique du français vers le tchèque, a montré que les professionnels ayant suivi une formation juridique atteignent de meilleurs résultats dans la manipulation du sens juridique et de la terminologique. À la lumière de cette constatation, la note globale semble moins paradoxale.

29. Cette différence est statistiquement significative (t-test unilatéral, $p=0,002$ ).

30. La méthodologie de cette évaluation diffère de la nôtre notamment en ce que les scores sont basés sur l'analyse d'endroits choisis («rich points»), et que la moitié des traducteurs n'avaient pas d'expérience en traduction non native. Les langues testées sont l'espagnol d'un côté, et l'anglais, le français et l'allemand de l'autre.

\section{RÉFÉRENCES}

AdAB, Beverly (2005): Translating into a Second Language: Can We, Should We? In: Gunilla Anderman et Margaret Rogers, dir. In and Out of English: For Better, for Worse? Buffalo: Multilingual Matters, 227-241.

BAorong, Wang (2009): The Issue of Direction of Translation in China: A Historical Overview. Translation Journal. 13(3). Consulté le $1^{\text {er }}$ mai 2017, $<$ http://translationjournal.net/journal/49direction.htm>. 
Beeby Lonsdale, Allison (2009): Directionality. In: Mona Baker et Gabriela Saldanha, dir. Routledge Encyclopedia of Translation Studies. London/New York: Routledge, 84-88.

CAmpbell, Stuart (1998): Translation into the Second Language. New York: Longman.

CAmpbell, Stuart (2005): English Translation and Linguistic Hegemony in the Global Era. In: Gunilla Anderman et Margaret Rogers, dir. In and Out of English: For Better, for Worse? Buffalo: Multilingual Matters, 27-38.

Crystal, David (2003): English as a Global Language. Cambridge: Cambridge University Press.

DubĚDA, Tomáš et OBDRŽÁlková, Vanda (2018): Terminologická a textová kompetence v právním překladu: studie na francouzsko-českém materiálu [La compétence terminologique et textuelle dans la traduction juridique: analyse de traductions franco-tchèques]. Studie $z$ aplikovane lingvistiky. 2018(1):73-91.

Grosjean, François et Dommergues, Jean-Yves (2011): La statistique en clair. Paris: Ellipses.

Hansen, Gyde, et al. (1998): The translation process: from source text to target text. In: Gyde HANSEN, dir. LSP texts and the process of translation. Copenhagen working papers in LSP. Copenhagen: Samfundslitteratur, 59-72.

Hurtado Albir, Amparo, dir. (2017): Researching Translation Competence by PACTE Group. Amsterdam/Philadephie: John Benjamins.

Kelly, Dorothy, Nobs, Marie-Louise, SÁnChez, Dolores et WAY, Catherine (2006): Reflections on Directionality in Translator Training. Forum. 4(1):57-81.

Martínez Mateo, Roberto (2014) : A deeper look into metrics for translation quality assessment (TQA): A case study. Miscelánea: A Journal of English and American Studies. 49:73-94.

McAlester, Gerard (1992): Teaching translation into a foreign language - status, scope and aims. In: Cay Dollerup et Anne LoDDEGA ARD, dir. Teaching Translation and Interpreting. Amsterdam/Philadelphie: John Benjamins, 291-297.

Mossop, Brian (2001): Revising and Editing for Translators. Manchester: St. Jerome Publishing.

Newmark, Peter (1988): Approaches to Translation. London: Prentice Hall.

OBDRŽÁLKovÁ, Vanda (2016): Translation as a decision-making process: an application of the model proposed by Jiří Levý to translation into a non-mother tongue. Mutatis mutandis. 9(2):306-327.

O'BrIEN, Sharon (2012): Towards a dynamic quality evaluation model for translation. Journal of Specialised Translation. 17:55-77.

Pavlović, Nataša (2007): Directionality in translation and interpreting practice. Report on a questionnaire survey in Croatia. Forum. 5(2):79-99.

РокоRn, Nike (2005): Challenging the Traditional Axioms. Translation into a non-mother tongue. Amsterdam/Philadelphie: John Benjamins.

Prieto Ramos, Fernando (2015): Quality Assurance in Legal Translation: Evaluating Process, Competence and Product in the Pursuit of Adequacy. International Journal for the Semiotics of Law. 28(11):11-30.

PRUNČ, Erich (2000) : Translation in die Nicht-Muttersprache und Translationskultur [Traduction dans sa langue maternelle et sa propre culture de traduction]. In: Meta Grosman, Mira KADRIĆ, Irena KovačIČ et Mary SNELL-Hornby, dir. Translation into Non-Mother Tongues: In Professional Practice and Training. Tubingue: Stauffenburg, 5-20.

Roiss, Silvia (2001): El Mercado de la traducción inversa en España. Un estudio estadístico. Hermeneus. Revista de traducción e interpretación. 3:379-408.

Snell, Barbara et Crampton, Patricia (1989): Types of translation. In: Catriona Picken, dir. The Translator's Handbook. Londres: Aslib, 59-70.

Taviano, Stefania (2013): English as a Lingua Franca and Translation. Implications for Translator and Interpreter Education. The Interpreter and Translator Trainer. 7(2):155-167.

Thelen, Marcel (2005): Translating into English as a Non-native Language: The Dutch Connection. In: Gunilla Anderman et Margaret Rogers, dir. In and Out of English: For Better, for Worse? Buffalo: Multilingual Matters, 242-255.

Toury, Gideon (1995): Descriptive Translation Studies and Beyond. Amsterdam/Philadelphie: John Benjamins. 
WAGNER, Emma (2005): Translation and/or Editing - The Way Forward? In: Gunilla ANDERMAN et Margaret Rogers, dir. In and Out of English: For Better, for Worse? Buffalo: Multilingual Matters, 214-226.

Wimmer, Stefanie (2011) : El Proceso de la Traducción Especializada Inversa. Thèse de doctorat non publiée. Barcelone: Universitat Autònoma de Barcelona.

\section{RESSOURCES WEB}

FilozofickÁ FakUlta [Faculté de philosophie] (17 février 2018): Studijní plány [Cursus offerts]. Univerzita Karlova [Université Charles de Prague]. Consulté le 5 mai 2018, <https://www. ff.cuni.cz/studium/studijni-obory-plany/studijni-plany/>.

Parlament České republiky [Parlement de la République tchèque] (6 avril 1967): Zákon č. 36 o znalcích a tlumočnících [Loi no. 36 relative aux experts et interprètes assermentés]. Consulté le 3 mai 2018, <https:// www.kstcr.cz/cz/legislativa-zakony-zakon-c-36-1967-sbo-znalcich-a-tlumocnicich-v-platnem-zneni>.

Parlament České Republiky [Parlement de la République tchèque] (3 février 2012): Občanský zákoník [Code civil]. Ministerstvo spravedlnosti České republiky [Ministère de la justice de la République tchèque]. Consulté le 3 mai 2018, <http://obcanskyzakonik.justice.cz/images/ pdf/Code-civil.pdf $>$.

Syndicat national des traducteurs Professionnels (2011): Traduction. Faire les bons choix. Petit guide de l'acheteur de traductions. Société française des traducteurs. Consulté le $1^{\text {er }}$ mai 2017, <https://www.sft.fr/clients/sft/telechargements/file_front/4c10e27819e7f.pdf>.

UNESCO (1976) : Recommandation sur la protection juridique des traducteurs et des traductions et sur les moyens pratiques d'améliorer la condition des traducteurs. Consulté le $1^{\text {er }}$ mai 2017,

<http://portal.unesco.org/fr/ev.php-URL_ID=13089\&URL_DO=DO_TOPIC\&URL_SECTION=201.html $>$.

\section{ANNEXES}

\section{Annexe 1 : Exemples des textes de départ}

Texte publicitaire, FR $>C S$

Spécialisée en fabrication de cuisines sur mesure, l'entreprise familiale Gilles Parois Cuisines se situe à Saint-Jean-de-Boiseau (44) proche de Nantes.

M. Parois Gilles, père des deux co-gérants actuels a fondé cette entreprise dans les années 70. Reprise ensuite par ses deux fils Mickaël et Stéphane, passionnés par le métier de cuisiniste, la société s'adresse essentiellement aux particuliers. [...]

Différents matériaux sont envisageables pour la réalisation de vos meubles. Lors de votre visite, nous vous ferons part d'une liste exhaustive dans laquelle vous pourrez choisir le bois ou la matière adaptée à vos envies. En tant que fabriquant nous avons la possibilité de proposer des aménagements personnalisés.

Pour une bonne préparation du chantier nous faisons appel selon le souhait du client aux artisans avec lequel nous travaillons dans le domaine de l'électricité et de la plomberie. Retrouvez toutes nos cuisines en images dans la suite du site et inspirez-vous des photos de notre galerie pour vos propres projets!

\section{Texte juridique, EN>CS}

The Photographer is the Author of the photograph.

The Client is the person or organisation to whom the invoice is addressed (whether or not the Client is acting for a third party).

The License to Use only comes into effect once full payment of the invoice has been made. 
No use may be made of the images until full and final payment - including any late payment charges that may have been levied - has been received by the Company.

Permission in writing may be granted for image use before payment, however this permission will be immediately revoked if payment of the invoice is not made by the timescale stated on the invoice.

Reproduction rights (if and when granted) are strictly limited to the use and period of time specified on the Company's invoice. An agreement must be reached with the Company before the pictures are used for a different purpose or after the licence to use has expired. $[\ldots]$

\section{Texte juridique, CS>FR, EN}

Není-li dohodnuto něco jiného, je služba poskytována tak, že klient předá provozovateli prostřednictvím rozhraní serveru či jiným dohodnutým způsobem obchodní sdělení ve formátu vyžadovaném provozovatelem a provozovatel či jím pověřená osoba rozešlou obchodní sdělení vybraným adresátům, a to pod jménem klienta.

O rozeslání obchodního sdělení informuje provozovatel klienta prostřednictvím rozhraní serveru, nedohodnou-li se klient a provozovatel jinak.

$\mathrm{V}$ př́padě, že provozovateli vznikne škoda v důsledku porušení těchto Podmínek a/nebo zákona uživatelem v souvislosti s užíváním serveru a jeho služeb, s daty zadanými uživatelem pro zařazení do databáze a s daty poskytnutými klienty k rozeslání ve formě obchodního sdělení adresátům, zavazuje se př́ílušný uživatel takovou škodu uhradit za provozovatele, a není-li to možné, nahradit takovou škodu provozovateli následně v plné výši.

Odvolání souhlasu registrovaného uživatele se zpracováním jeho osobních údajů má obvykle za následek automatické odebrání registrace. [...]

\section{Annexe 2: Questionnaires}

Seules les questions pertinentes pour la présente étude sont reproduites.

Questionnaire 1 (à remplir avant la traduction)

- Question 3: Pour la paire de langues en question, quel est le pourcentage de textes que vous traduisez vers la langue étrangère? Chosissez une des options suivantes: 0-20\%; $21-40 \%$; 41-60\%; 61-80\%; 81-100\% (Les étudiants sautent cette question.)

- Question 4: Pour la paire de langues en question, estimez-vous que la traduction vers le tchèque et la traduction vers la langue étrangère diffèrent quant à leur difficulté? Chosissez une des options suivantes: la traduction vers la langue étrangère est plus difficile; la traduction vers le tchèque est plus difficile; les deux directions ont la même difficulté.

- Question 16: Quels cours ou formations ayant un rapport avec la traduction avez-vous suivi en dehors de vos études universitaires en traduction?

Questionnaires 2 et 3 (à remplir après la traduction des textes publicitaire et juridique, respectivement)

- Question 6: Quelle est la fréquence avec laquelle vous traduisez ce type de textes (présentation d'une entreprise/conditions d'utilisation d'un site web)? Chosissez une des options suivantes: jamais; quasiment jamais; parfois; souvent.

- Question 7: Êtes-vous content(e) de la traduction que vous venez de finir? Chosissez une des options suivantes: très content(e); plutôt content(e); ni content(e) ni mécontent(e); plutôt mécontent(e); très mécontent(e). 